\title{
EEG Verileri Kullanılarak Fiziksel El Hareketleri ve Bu Hareketlerin Hayalinin Yapay Sinir Ağları İle Sınıflandırılması
}

\author{
Mustafa TOSUN; Corresponding Author; mustafa.tosun@dpu.edu.tr \\ Kütahya Dumlupınar Üniversitesi \\ Simav Teknoloji Fakültesi Elektrik Elektronik Mühendisliği \\ Mustafa ERGINLI; Corresponding Author; mustafa.erginli@dpu.edu.tr \\ Kütahya Dumlupınar Üniversitesi \\ Simav Meslek Yüksekokulu \\ Ömer KASIM; omer.kasim@dpu.edu.tr \\ Kütahya Dumlupınar Üniversitesi \\ Simav Teknoloji Fakültesi Elektrik Elektronik Mühendisliği \\ Burak UĞRAŞ; burak.ugras@ogr.dpu.edu.tr \\ Kütahya Dumlupinar Üniversitesi \\ Simav Teknoloji Fakültesi Elektrik Elektronik Mühendisliği \\ Şems TANRIVERDİ; sems.tanriverdi1905@gmail.com \\ Kütahya Dumlupınar Üniversitesi \\ Simav Teknoloji Fakültesi Elektrik Elektronik Mühendisliği \\ Tayfun KAVAK; tayfunnkavak@hotmail.com \\ Kütahya Dumlupınar Üniversitesi \\ Simav Teknoloji Fakültesi Elektrik Elektronik Mühendisliği
}

Received 15 July 2018; Accepted 21 July 2018; Published online 03 August 2018

\section{Özet}

Son yillarda teknolojinin gelişmesi sonucunda beyin bilgisayar arayüzü ile ilgili çalışmalar artmıştır. Beyin Bilgisayar Arayüzü (Brain Computer Interface-BCI) yöntemlerinde Elektroansefalogram (ElectroencephalogramEEG) işaretleri yaygın olarak kullanılmaktadır. EEG verileri kullanılarak fiziksel hareketle hareketin hayali sınıflandırılabilmektedir. Bu çalışmada sağ elini kullanan ve hastalık durumu olmayan 21 yaşındaki bir erkeğe ait EEG verileri kullanılmıştır. Bu verilerin bir kısmı sol ve sağ elin ileri-geri hareketi esnasında kaydedilen EEG verileridir. Diğer veriler ise herhangi bir fiziksel hareket yapılmadan, hareketin hayal edilmesi durumu ile ilgili kayıtlardır. Welch metodu kullanılarak EEG verilerinin 1-48 Hz arasındaki frekanslarının güç yoğunlukları hesaplanmıştır. Elde edilen veri setleri tasarlanan Geri Yayılımlı Sinir Ağ 1 (Backpropagation Neural NetworkBPNN) ' na uygulanmıştır. A ğın eğitimi sonunda 4.6731 $\times 10^{-23}$ ortalama karesel hata (Mean Squared Error -MSE) değerine ulaşılmıştır. Hayal ile hareket verilerinden oluşan test veri seti eğitilen ağa uygulandığında, hayal ile hareket verileri \% 99.9975 doğrulukla sınıflandırılmıştır.

\begin{abstract}
In recent years, as a result of the technological development, there has been a significance improvement on the computer interface. Electroencephalogram (EEG) signals are widely used in Brain Computer Interface (BCI) methods. By using EEG data, the imagination of movement with physical motion can be classified. In this study, EEG data of a 21-years-old man who used his right hand and who didn't show any disease symptom was used. Part of this EEG data demonstrates the recordings of forward and backward movement of the left and right hand. The other data indicates the records of imagination of motion without any physical movement. Using the Welch method, the power densities of the frequencies of 1-48 Hz of the EEG data were calculated. The obtained data sets were applied to the designed Back Propagation Neural Network (BPNN). At the end of the network training, the Mean Squared Error (MSE) value of 4.6731x10-23 has been reached. When the test data set, which consists of imaginary and motion data is applied to the trained network, imagination and motion data are classified with accuracy of $99.9975 \%$.
\end{abstract}

Keywords: EEG, backpropagation neural network, welch method, signal processing, EEG classification 


\section{Giriş}

Beyin Bilgisayar Arayüzü (Brain Computer Interface- BCI), bireyin dış dünyaya gönderdiği mesajların veya komutların, beynin periferal sinirler ve kasların normal çıkış yollarından geçmediği bir iletişim sistemidir [1]. BCI teknolojisi, kullanıcılar ve sistemler arasında güçlü bir iletişim aracıdır [2]. BCI sistemi beyin sinyallerini kaydeder ve istenen görevi tamamlamak için bunları bilgisayar sistemine gönderir[3].

Elektroansefalogram (Electroencephalogram-EEG) işaretleri diğer cihazlardan elde edilen işaretlere oranla yüksek çözünürlüğe sahip olması nedeniyle BCI çalışmalarında yaygın olarak kullanılmaktadır[4].

Kafa derisinin yüzeyinden ölçülen EEG potansiyelleri, beynin sinirsel aktivitesinin sonucu olarak elde edilen biyoelektrik sinyallerdir. Elektroensefalogram (EEG) işaretleri, serebral korteksin çok geniş bir alanından ve birçok alt noktadan gelen potansiyellerin toplamıdır [5].

EEG analizlerinde çeşitli frekans bantları kullanılmaktadır. Bu bantlar delta- $\delta(1-3 \mathrm{~Hz})$, teta- $\theta(4-7 \mathrm{~Hz})$, alfa- $\alpha(8-13 \mathrm{~Hz})$ ve beta- $\beta(13-30 \mathrm{~Hz})$ olarak sinıflandırılmaktadır [6]. EEG verilerine Hızlı Fourier dönüşümü (FFT) uygulanarak frekans boyutunda analizler yapılabilir. EEG verilerinin analizinde ayrıca Dalgacık dönüşümü [7], Hilbert-Huang dönüşümü [8], Fast Walsh Hadamard dönüşümü (FWHT) [9] ve güç yoğunluğu parametreleri [10] de kullanılmaktadır.

Do ve arkadaşları, EEG sinyallerinin analizi ile Fonksiyonel Elektrik Uyarma Sistemi (Functional Electrical Stimulation System) adı verilen ve ayak hareketlerini yitirmiş hastaların ayak hareketlerini yönetebilen sistem ile ilk başarılı uygulamayı gerçekleştirmişlerdir [11]. Mabrouk, kullanıcının EEG sinyallerini görüntüleyen ve bunları yüksek doğrulukla hayali sağ ve sol el hareketleri olarak sinıflandıran EEG tabanlı-BCI sistemi geliştirmiştir [12]. C. Liu ve arkadaşları, sağ el hareketinin hayali ile sol el hareketinin hayalini C3 / C4 ve P3 / P4 kanallarına ait EEG verilerinin ortalama güçlerini kullanarak sınıflandırmışlardır. Kesikli Dalgacık Dönüşümü ve Fisher Doğrusal Ayrımı Analizi yöntemlerini kullanarak \%75.5 doğrulukla, Kesikli Dalgacık Dönüşümü ve Destek Vektör Makinası yöntemlerini kullanarak \% 82.1 doğrulukla sınıflandırma yapmışlardır [13]. Robinson ve Vinod, yaptıkları çalışmada kişinin baskın eli ile ilgili iki yönlü hareket ve bu hareketlerin hayali arasında sınıflandırma yapmışlardır. İki yönlü hareketi \%58.7 doğrulukla, hayali ise \%57.7 doğrulukla sınıflandırmışlardır [14]. Hajibabazadeh ve Azimirad, 6 Kanaldan topladıkları EEG verilerini filtreleyerek Dalgacık dönüşümü yöntemi ile özellik çıkarma işlemini gerçekleştirmişlerdir. Sağ el ve sol el hareketlerini sınıflandırmada Destek vektör makine yöntemini kullanarak \% 75 başarı elde etmişlerdir [15].

Bu çalışmada sağ elini kullanan ve hastalık durumu olmayan 21 yaşındaki bir erkeğe ait EEG verileri kullanılmıştır. EEG verileri gözler kapalı olarak kaydedilmiştir. Bu verilerin bir kısmı sol ve sağ elin ileri-geri hareketleri iken diğer kayıtlar ise herhangi bir fiziksel hareket yapılmadan, hareketin hayal edilmesi durumu ile ilgili kayitlardır. EEG kaytları FP1, FP2, F3, F4, C3, C4, P3, P4, O1, O2, F7, F8, T3, T4, T5, T6, Fz, Cz, Pz elektrotlarından alınmıştır. EEG kaydı için örnekleme frekans1 $500 \mathrm{~Hz}$ olan Neurofax EEG Sistemi kullanılmıştır. Welch metodu kullanılarak 19 kanala ait EEG verilerinin 1-48 $\mathrm{Hz}$ arasındaki frekanslarının güç yoğunlukları hesaplanmıştır. Elde edilen veri setleri tasarlanan Geri Yayılmalı Sinir Ağı (Backpropagation Neural Network- BPNN)' na uygulanmıştır. Ağın eğitimi sonunda $4.6731 \times 10^{-23}$ ortalama karesel hata (Mean Squared Error -MSE) değerine ulaşılmıştır. Hayal ile hareket verilerinden oluşan test veri seti eğitilen ağa uygulandığında, hayal ile hareket verileri \% 99.9975 doğrulukla sınıflandırılmıştır.

\section{Materyal ve Metot}

Yapay Sinir Ağları insan beyninin çalışma ve öğrenebilme yeteneğinden yola çıkılarak oluşturulmuş bir bilgi işlem teknolojisidir [16]. Yapay sinir ağları ile ilgili birçok algoritma geliştirilmiş ve pek çok alanda kullanılmıştır. Bu çalışmada Geri Yayılmalı Sinir Ağı algoritması ile EEG sinyallerinin sınıflandırılması yapılmıştır. 


\subsection{Geri Yayılmalı Sinir A $\breve{g} ı$}

Yapay sinir ağlarında parametrelerin güncellenmesinde sık kullanılan yöntemlerin başında geri yayılma algoritmaları gelmektedir. Genellenmiş delta kuralı, geri yayılmalı ăg eğitilirken hedef değerler ile çıkış değerleri arasındaki farkın (hata) bir kısmı, her bir eğitim hücresine geri yansıtılarak ağırlıkların hataya göre değiştirilmesini ve bu işlemin belli sayıda tekrarlanarak hatanın en küçük değere ulaşmasını sağlayan bir yöntemdir [6]. Geri yayılmalı ağ yapısı Şekil 1 'de gösterilmiştir.

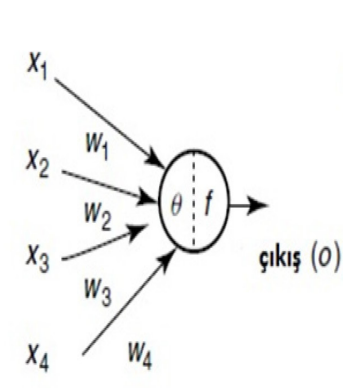

(a) Yapay nöron

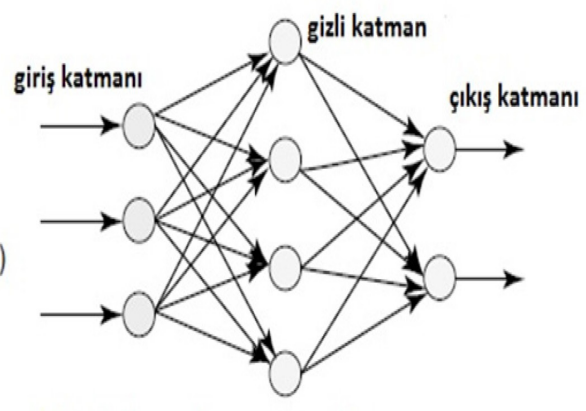

(b) Çok katmanlı yapay sinir ağı

Şekil 1 Çok Katmanlı A $\breve{g}$ Yapısı[17]

Giriş vektörü $X_{p}=\left(x_{p 1}, x_{p 2}, \ldots . x_{p n}\right)$ yapay sinir ağının giriş katmanına uygulandığında j'inci ara birim için ağ girişi eşitlik 1 de verilmiştir.

$$
n e t_{p j}^{h}=\sum_{i=1}^{N} W_{j i}^{h} X_{p i}+\theta_{j}^{h}
$$

Eşitlik 1'de $W_{j i}^{h}, i$ ' inci giriş biriminden $j$ ' inci ara birime olan bağlantı üzerindeki ağırlıktır. Ara katman üzerindeki nicelikler h ile tanımlanmıştır. Çıkış birimleri için "o" kullanılmıştır.

$$
n e t_{p k}^{o}=\sum_{j=1}^{L} W_{k j}^{0} I_{p j}+\theta_{k}^{0}
$$

Eşitlik 1 ve eşitlik 2'de gösterilen $\theta$ terimi bias terimidir. +0.5 ile -0.5 arasında bir değer ile tanımlanabilir.

$$
\delta_{p k}=\left(y_{p k}-\boldsymbol{O}_{p k}\right)
$$

Yapay sinir ağında hata değeri, eşitlik 3 ile hesaplanır. $\delta_{p k}$, p'inci eğitim vektörünün k'ıncı çıkışındaki hata değeridir. $y_{p k}$, istenilen çıkış, $O_{p k}$ ise o anda yapay sinir ağının hesapladığı çıkıştır. Tüm çıkış birimlerinin hatalarının karelerinin toplamı eşitlik 4 ve eşitlik 5 'te verilmiştir. 


$$
\begin{aligned}
& E_{p}=\frac{1}{2} \sum_{k=1}^{M} \delta_{p k}^{2} \\
& E_{p}=\frac{1}{2} \sum_{k}\left(y_{p k}-O_{p k}\right)^{2}
\end{aligned}
$$

\subsection{EEG Sinyal İşleme}

EEG sinyallerinden elde edilen en yaygın özellikler spektral özelliklerdir[18]. Spektral kestirim için parametrik olmayan yöntemler en genel spektral analizlerdir. Bu analizler için genellikle Fourier dönüşümüne dayanan Hızlı Fourier dönüşümü (FFT) algoritması uygulanır. Welch metodu (veya segment ortalaması), iyi bilinen bir parametrik olmayan güç spektral yoğunluk (PSD) tahmin edicisidir [19]. Welch metodu aşağıdaki eşitliklerde verilmiştir.

$$
x_{i}(n)=x(n+i D), n=0,1,2, \ldots,(M-1)
$$

Eşitlik 6' da $x_{\mathrm{i}}$ veri dizisini, iD dizinin başlangıç noktasını, $\mathrm{M}$ oluşturulmuş veri bölümlerini göstermektedir. Periyodgramları hesaplamak için eşitlik 7 kullanılmaktadır [20]. Burada verilen U, gücün normalizasyonudur ve eşitlik 8' de verilmiştir.

$$
\begin{aligned}
& P_{\mathrm{xx}} \quad(\mathbf{f})=\frac{1}{M U}\left(\sum_{n=0}^{M-1} \mathrm{x}_{\mathrm{i}}(\mathrm{n}) \mathrm{w}(\mathrm{n}) \mathrm{e}^{-\mathrm{j} 2 \pi \mathrm{fn}}\right)^{2} \\
& U=\frac{1}{M} \sum_{n=0}^{M-1} W^{2}(n)
\end{aligned}
$$

W(n) pencere fonksiyonudur. Değiştirilmiş periodogramların ortalaması Welch'in güç spektrumunun verir. Güç spektrumu eşitlik 9' da gösterilmiş̧ir [20, 21].

$$
\mathrm{P}_{\mathrm{xx}}^{\mathrm{W}}=\frac{1}{\mathrm{~L}} \sum_{\mathrm{i}=0}^{\mathrm{L}-1} \approx(\mathrm{i})
$$

Burada L, uzunluğu göstermektedir.

Bu çalışmada Brain Computer Interface Research at NUST Pakistan'a ait web sitesindeki açık kaynak olarak sunulan Project BCI - EEG Motor Activity Veri Seti içindeki Veri Seti 2 verileri kullanılmıştır [22]. Bu veriler, sağ elini kullanan ve hastalık durumu olmayan 21 yaşındaki bir erkeğe aittir. EEG verilerinin bir kısmı sol ve sağ elin ileri-geri hareketleri esnasında gözler kapalı olarak kaydedilmiştir. Diğer kayıtlar ise herhangi bir fiziksel hareket yapılmadan, hareketin hayal edilmesi durumu ile ilgili kayıtlardır. EEG kayıtları FP1, FP2, F3, F4, C3, C4, P3, P4, O1, O2, F7, F8, T3, T4, T5, T6, Fz, Cz, Pz 
elektrotlarından alınmıştır. Kullanılan elektrotların açıklamaları Tablo 1' de verilmiştir. Elektrotların kafa derisi üzerindeki yerleşimi Şekil 2' de gösterilmiştir. EEG kaydı için örnekleme frekansı $500 \mathrm{~Hz}$ olan Neurofax EEG Sistemi kullanılmıştır. AC sinyallerin gürültüsünden etkilenmemesi için $50 \mathrm{~Hz}$ ' lik Notch filtre kullanılmıştır.

Tablo 1 EEG' de kullanılan elektrotların kısaltma ve açıklamaları

\begin{tabular}{ll}
\hline Elektrot Kısaltmaları & Açılamaları \\
\hline FP & Frontopolar \\
F & Frontal \\
P & Parietal \\
C & Central \\
T & Temporal \\
O & Occipital \\
Z & Midline
\end{tabular}

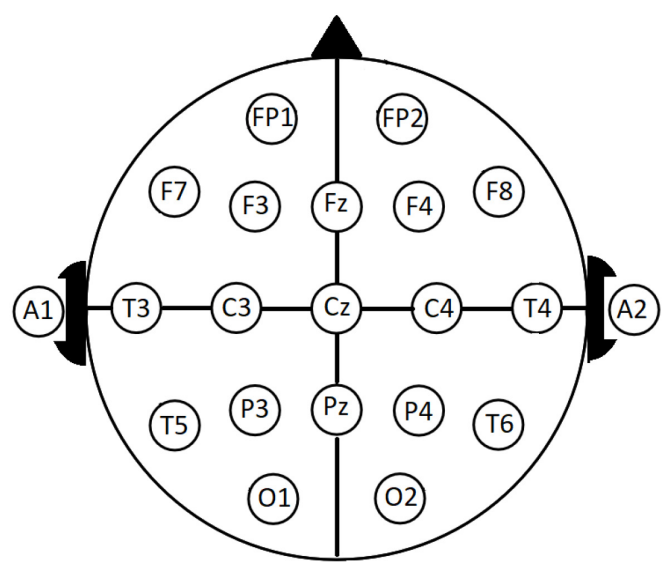

Şekil 2 Uluslararası 10-20 sistemine göre elektrotların kafa derisi üzerindeki yerleşimi

Tablo 2 EEG verilerinin içerikleri

\begin{tabular}{llll}
\hline El Durumu & Veri Tipi & Deneme Sayıs1 & Hareket Yönü \\
\hline Sol & Fiziksel & 3 & İleri \\
Sol & Hayal & 2 & İleri \\
Sol & Fiziksel & 3 & Geri \\
Sol & Hayal & 2 & Geri \\
Sağ & Fiziksel & 3 & İleri \\
Sağ & Hayal & 2 & İleri \\
Sağ & Fiziksel & 3 & Geri \\
Sağ & Hayal & 2 & Geri
\end{tabular}

Tablo 2 de sağ el, sol el, hareket yönleri, deneme sayıları ve hareketin hayal ya da gerçek olma durumlarında kaydedilen EEG verileriyle ilgili bilgiler verilmiştir [22]. Fiziksel hareketler esnasında kaydedilen veriler 6 saniyelik zaman diliminde, hareketin hayal edilmesi durumunda kaydedilen veriler ise 14 saniyelik zaman diliminde alınmıştır. Böylece fiziksel hareketlerin her bir denemesinde $3008 \mathrm{x}$ 19 adet veri, hareketin hayal edilmesi denemelerinde ise 7040x19 adet veri elde edilmiştir. Hayal denemeleriyle ilgili veriler bölünerek iki ayrı hayal verisi olarak kullanılmıştır.

Welch metodu kullanılarak 19 kanala ait EEG verilerinin 1-48 Hz arasındaki frekanslarının güç yoğunlukları hesaplanmıştır. Elde edilen güç yoğunluklarının ortalamaları alınarak 19 x 1 adet veri elde edilmiştir. Sonuç olarak 19 × 20 adet güç yoğunluk verisi elde edilmiştir. Bu verilerden fiziksel kol 
hareket verilerine ait 12 adet veri setinin grafiği Şekil 3' de gösterilmiştir. Şekil 4' de ise hayal edilen kol hareket verilerine ait 8 adet veri setinin grafiği verilmiştir.

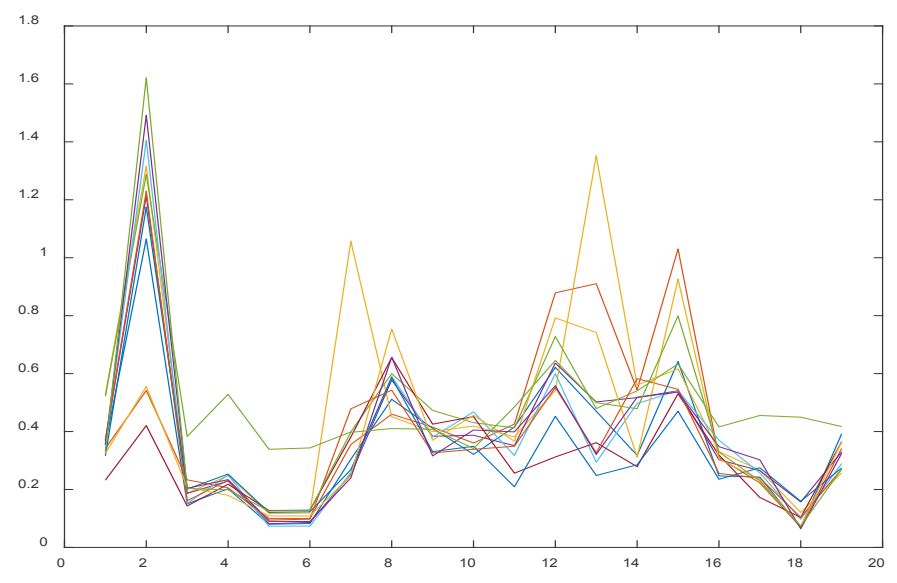

Şekil $3 \mathrm{Kol}$ hareket verilerine ait 12 adet veri seti grafiği

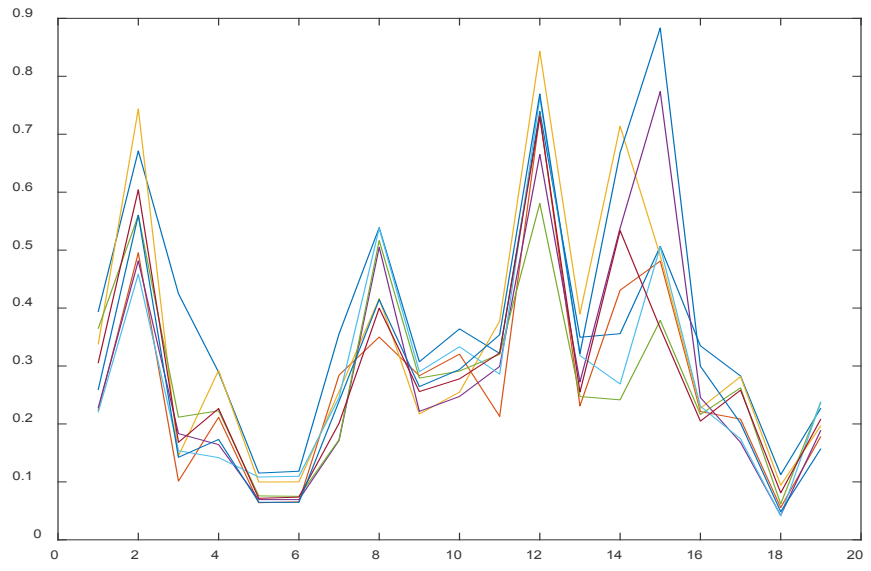

Şekil 4 Hayal edilen kol hareket verilerine ait 8 adet veri setinin grafiği

Güç yoğunluk verilerinden $19 \times 12$ adet veri eğitim seti için, 19 × 8 adet veri ise test seti için ayrılmıştır. Eğitim ve test veri setleri 19 kanaldan elde edilen EEG verilerinin frekanslarına ait ortalama güç yoğunluk değerlerinden oluşmaktadır. Eğitim veri seti aşırı uyumu (overfitting) ve yetersiz uyumu (underfitting) engellemek için 19x12 adet eğitim verisi yan yana iki defa birleştirilerek çoğaltılmıştır. Sonuç olarak 19 x 36 adet eğitim verisi elde edilmiştir. Böylelikle her biri 19 adet öznitelik verisinden oluşan 36 adet girdi vektörü ağa uygulanmıştır. Eğitim setindeki her bir veri örneğine karşılık gelen hedef çıktılar için 1x36 adet veri içeren hedef seti oluşturulmuştur. Bu hedef seti, eğitim setindeki fiziksel hareket örnek verileri için 0.1 çıktı değerlerini, hayal edilen hareket örnekleri için 0.9 çıktı değerlerini içermektedir.

Elde edilen veri setleri için BPNN ağ modeli tasarlanmıştır. A $\breve{g}$ mimarisi girdi katmanı, 1 adet gizli katman ve çıktı katmanından oluşturulmuştur. Girdi katmanında 19 adet nöron, gizli katmanda 19 nöron ve çıktı katmanında ise 1 adet nöron kullanılmıştır. Ağ eğitimi için oluşturulan eğitim verileri, tasarlanan ağ tarafından eğitime başlamadan önce, rastgele olarak eğitim, test ve doğrulama setlerine ayrılmıştır.

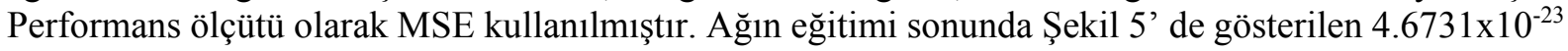
performans değerine (MSE) ulaşılmıştır. 


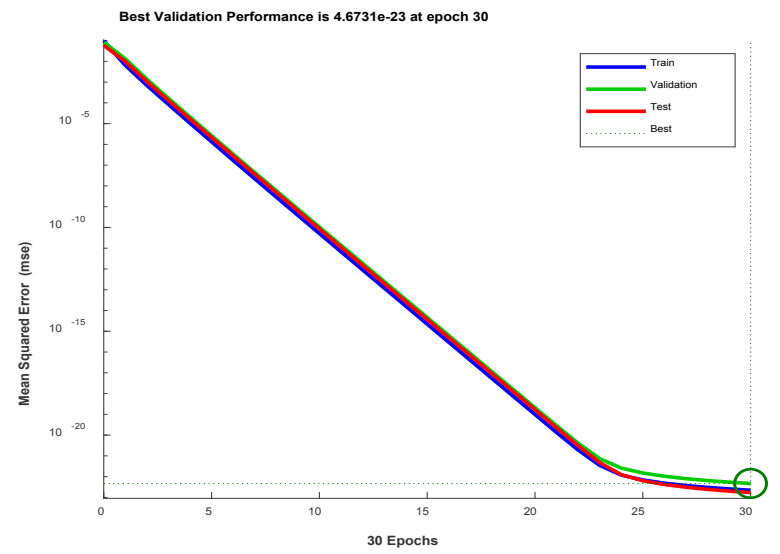

Şekil 5 Tasarlanan ağın performans (MSE) grafiği

\section{Sonuçlar ve Tartışma}

Elde edilen veri setleri tasarlanan BPNN ağına uygulandığında $4.6731 \times 10^{-23}$ performans değerine (MSE) ulaşılmıştır. Simülasyon sonuç verileri ve hedef test verileri Tablo 3' de verilmiştir. Burada hayal ile hareket verilerinin \% 99.9975 doğrulukla sınıflandırıldığı görülmektedir.

$\mathrm{Bu}$ çalışmanın sonucunda Welch metodu ile elde edilen EEG sinyallerinin güç spektrum yoğunluk değerleri, BPNN ağı ile sınıflandırıldığında fiziksel el hareketi ile bu hareketin hayalinin yüksek doğruluk oranıyla birbirinden ayırt edilebildiği görülmüştür.

Elde edilen sonuçlar ellerini veya kollarını yitirmiş bireyler için implant teknolojilerinde kullanılabilmesi bakımından önemlidir. Ayrıca BCI uygulamalarında hareketlerin hayal edilmesi ile robotik sistemler düşünce ile kontrol edilebilir.

Bu çalışmada bir kişiden elde edilen veriler kullanılarak hareket ile hareketin hayalinin sınıflandırılması yapılmıştır. Daha sonraki çalışmalarda farklı yaşlardaki kadın ve erkek birden fazla kişinin verileri kullanılarak sağ ve sol elin ayrı ayrı hareket ve hayalinin ayrımı, sağ ayak ve sol ayağın hareket ve hayalinin ayrımı çalışmaları yapılabilir.

Tablo 3 Simülasyon ve Hedef Test Verileri

\begin{tabular}{ll}
\hline $\begin{array}{l}\text { Test Simülasyonu } \\
\text { Sonuç Verileri }\end{array}$ & Test Hedef Verileri \\
\hline 0,100000086919240 & 0,100000000000000 \\
0,100000001309471 & 0,100000000000000 \\
0,100000000114541 & 0,100000000000000 \\
0,100000000015895 & 0,100000000000000 \\
0,899998615198394 & 0,900000000000000 \\
0,899902924319162 & 0,900000000000000 \\
0,899999999996019 & 0,900000000000000 \\
0,899999999980233 & 0,900000000000000
\end{tabular}

\section{Etik Durum}


Bu çalışmada Brain Computer Interface Research at NUST Pakistan' a ait web sitesindeki açık kaynak olarak sunulan Project BCI - EEG Motor Activity Veri Seti içindeki Veri Seti 2 verileri kullanılmıştır[22].

\section{Teşekkür}

EEG verilerini açık kaynak olarak kullanıma izin veren Brain Computer Interface Research at NUST Pakistan Kurumuna teşekkür ederiz. Ayrıca Kütahya Dumlupınar Üniversitesi, Simav Teknoloji Fakültesi, Elektrik-Elektronik Mühendisliği Bölümü ile yapılan protokol çerçevesinde, Biyofizik Anabilim Dalı Laboratuvarlarındaki MATLAB program çözümleriyle çalışmamıza izin veren Dokuz Eylül Üniversitesi Tıp Fakültesi Biyofizik Anabilim Dalı Başkanı ve yetkililerine teşekkür ederiz.

\section{References}

[1] J. R. Wolpaw, N. Birbaumer, D. J. McFarland, G. Pfurtscheller, and T. M. Vaughan, "Braincomputer interfaces for communication and control," Clinical Neurophysiology, vol. 113, no. 6 , pp. 767-791, 2002/06/01/ 2002.

[2] J. B. F. Van Erp, F. Lotte, and M. Tangermann, "Brain-computer interfaces: Beyond medical applications," Computer, Article vol. 45, no. 4, pp. 26-34, 2012, Art. no. 6165246.

[3] S. N. Abdulkader, A. Atia, and M.-S. M. Mostafa, "Brain computer interfacing: Applications and challenges," Egyptian Informatics Journal, vol. 16, no. 2, pp. 213-230, 2015/07/01/ 2015.

[4] R. A. Andersen, S. Musallam, and B. Pesaran, "Selecting the signals for a brain-machine interface," Current opinion in neurobiology, vol. 14, no. 6, pp. 720-726, 2004.

[5] E. Yazgan and M. Korürek, Tip elektroniği. İTÜ, 1996.

[6] M. Tosun and R. Güntürkün, "Anesthetic gas control with neuro-fuzzy system in anesthesia," Expert Systems with Applications, vol. 37, no. 3, pp. 2690-2695, 2010.

[7] S. K. Bashar, A. R. Hassan, and M. I. H. Bhuiyan, "Identification of motor imagery movements from eeg signals using dual tree complex wavelet transform," in Advances in Computing, Communications and Informatics (ICACCI), 2015 International Conference on, 2015, pp. 290296: IEEE.

[8] Y.-H. Liu, C.-A. Cheng, and H.-P. Huang, "Novel feature of the EEG based motor imagery BCI system: Degree of imagery," in System Science and Engineering (ICSSE), 2011 International Conference on, 2011, pp. 515-520: IEEE.

[9] K. Saka, Ö. Aydemir, and M. Öztürk, "Classification of EEG signals recorded during right/left hand movement imagery using Fast Walsh Hadamard Transform based features," in Telecommunications and Signal Processing (TSP), 2016 39th International Conference on, 2016, pp. 413-416: IEEE.

[10] S. Bhattacharyya, A. Khasnobish, A. Konar, D. Tibarewala, and A. K. Nagar, "Performance analysis of left/right hand movement classification from EEG signal by intelligent algorithms," in Computational Intelligence, Cognitive Algorithms, Mind, and Brain (CCMB), 2011 IEEE Symposium on, 2011, pp. 1-8: IEEE.

[11] A. H. Do, P. T. Wang, C. E. King, A. Abiri, and Z. Nenadic, "Brain-computer interface controlled functional electrical stimulation system for ankle movement," Journal of neuroengineering and rehabilitation, vol. 8, no. 1, p. 49, 2011.

[12] M. S. Mabrouk, "Non-Invasive EEG-based BCI system for Left or Right Hand Movement," Majlesi Journal of Electrical Engineering, vol. 5, no. 3, 2011.

[13] C. Liu, H. Wang, H. Pu, Y. Zhang, and L. Zou, "EEG feature extraction and pattern recognition during right and left hands motor imagery in brain-computer interface," in 2012 5th International Conference on BioMedical Engineering and Informatics, 2012, pp. 506-510.

[14] N. Robinson and A. P. Vinod, "Bi-Directional Imagined Hand Movement Classification Using Low Cost EEG-Based BCI," in 2015 IEEE International Conference on Systems, Man, and Cybernetics, 2015, pp. 3134-3139.

[15] M. Hajibabazadeh and V. Azimirad, "Brain-robot interface: Distinguishing left and right hand EEG signals through SVM," in 2014 Second RSI/ISM International Conference on Robotics and 
Mechatronics (ICRoM), 2014, pp. 813-816.

[16] E. Öztemel, "Yapay Sinir Ağlari," PapatyaYayincilik, Istanbul, 2003.

[17] A. Abraham, "129: Artificial Neural Networks," Handbook of Measuring System Design, 2005.

[18] M. Tosun, A. Ferikoğlu, R. Güntürkün, and C. Ünal, "Control of sevoflurane anesthetic agent via neural network using electroencephalogram signals during anesthesia," Journal of medical systems, vol. 36, no. 2, pp. 451-456, 2012.

[19] P. Welch, "The use of fast Fourier transform for the estimation of power spectra: a method based on time averaging over short, modified periodograms," IEEE Transactions on audio and electroacoustics, vol. 15, no. 2, pp. 70-73, 1967.

[20] A. S. Al-Fahoum and A. A. Al-Fraihat, "Methods of EEG signal features extraction using linear analysis in frequency and time-frequency domains," ISRN neuroscience, vol. 2014, 2014.

[21] J. G. Proakis and D. G. Manolakis, "Power spectrum estimation," Digital signal processing; principles, algorithms, and applications, 3rd edn. Prentice Hall, New Jersey, 1996.

[22] (15.06.2018). Project BCI - EEG Motor Activity Data Set. Available:

https://sites.google.com/site/projectbci/ 